\title{
Friction between Two Brownian Particles in a Lennard-Jones Solvent: A Molecular Dynamics Simulation Study
}

\author{
Song Hi Lee \\ Department of Chemistry, Kyungsung University, Busan 608-736, Korea.E-mail: shlee@ks.ac.kr \\ Received May 10, 2010, Accepted June 28, 2010
}

Key Words: Friction, Two Brownian particles, Lennard-Jones solvent, Molecular dynamics simulation

Molecular theory of Brownian motion of a single heavy particle in a fluid had received a considerable attention in earlier years. ${ }^{1-7}$ After molecular dynamics (MD) simulation technique was utilized, this subject has been widely studied by a variety of MD simulation methods. ${ }^{8-23}$ The common issues here were about the long time behavior of the force and velocity autocorrelation functions, the system size dependent friction coefficient of a massive Brownian particle, and test of the StokesEinstein law.

For the case of two heavy particles in a fluid, the generalized Langevin equation, obtained by Deutch and Oppenheim, ${ }^{24}$ resulted in a Fokker-Planck equation more general than the equation obtained by Mazo. ${ }^{25}$ For long times their result reduces to that found by Mazo. They evaluated the molecular expression obtained for the friction tensor by modification of an approximate hydrodynamic fluctuation theory argument developed by Zwanaig. ${ }^{26}$ The dependence of the friction tensors on inter-particle separations, $\mathrm{R}_{12}$, is obtained to the lowest order in $\left(a / \mathrm{R}_{12}\right)$ where $a$ is the radius of the Brownian particles.

Recently, The effects of hydrodynamic interactions on the friction tensors for two particles in solution were studied. ${ }^{27}$ The particles have linear dimensions on nanometer scales and are either simple spherical particles interacting with the solvent through repulsive Lennard-Jones forces or are composite cluster particles whose atomic components interact with the solvent through repulsive Lennard-Jones forces. The solvent dynamics is modelled at a mesoscopic level through multi-particle collisions that conserve mass, momentum and energy. ${ }^{28}$ The dependence of the two-particle relative friction tensors on the inter-particle separation indicated the importance of hydrodynamic interactions for these nano-particles.

In this work we focus on the calculation of the friction coefficients between two very massive Brownian particles in a Lennard-Jones solvent using MD algorithm of Newton's equations of motion. Our goal is to obtain the friction coefficient as function of the distance between the two Brownian particles and to compare with the hydrodynamic friction tensors evaluated by a molecular theory. ${ }^{24}$ This work is related to and motivated by our previous work ${ }^{22}$ in which equilibrium MD simulations in a microcanonical ensemble were performed to evaluate the friction coefficient of a Brownian particle (BP) in a LennardJones (LJ) solvent from the time dependent friction coefficients and the momentum autocorrelation functions of the BP with its infinite mass at various ratios of $\mathrm{LJ}$ parameters of the BP and solvent, $\sigma_{\mathrm{B}} / \sigma_{\mathrm{s}}$.

\section{Molecular Dynamics Simulation and Two-Particle Friction}

There are 20 Lennard-Jones (LJ) systems under investigation. Each system consists of two Brownian particles (BPs) with LJ parameter $\sigma_{\mathrm{B}}$ and solvent molecules of $\mathrm{N}=32,000$ each of which has LJ parameter $\sigma_{\mathrm{s}}$. The LJ potential used in our molecular dynamics (MD) simulations is a purely repulsive CWA (Chandler-Weeks-Andersen) potential: ${ }^{29}$

$$
\mathrm{v}^{\mathrm{CWA}}(\mathrm{r})=\left\{\begin{array}{lr}
4 \epsilon\left[\left(\frac{\sigma}{\mathrm{r}}\right)^{12}-\left(\frac{\sigma}{\mathrm{r}}\right)^{6}+\frac{1}{4}\right], & \mathrm{r} \leq \mathrm{r}_{\mathrm{c}} \\
0, & \mathrm{r}>\mathrm{r}_{\mathrm{c}}
\end{array}\right.
$$

where the LJ parameters are chosen as $\sigma=\left(\sigma_{\mathrm{B}}+\sigma_{\mathrm{s}}\right) / 2$ for the $\mathrm{BP}$-solvent molecule interaction or $\sigma=\sigma_{\mathrm{s}}$ for interaction between solvent molecules and $\varepsilon=1.006 \mathrm{~kJ} / \mathrm{mol}$. The inter-particle potential is truncated at $r_{c}=2^{1 / 6} \sigma$. The preliminary microcanonical ensemble (NVE fixed) molecular dynamics (MD) simulation for $\mathrm{N}=32,000 \mathrm{LJ}$ molecules of mass of $\mathrm{m}=3.9948 \mathrm{~g} / \mathrm{mol}$ was started in the cubic box of length $\mathrm{L}=6.84 \mathrm{~nm}$, of which the density is equal to $0.6633 \mathrm{~g} / \mathrm{cm}^{3}$. The corresponding reduced number density is $\rho^{*}=\rho \sigma_{\mathrm{s}}{ }^{3}=(\mathrm{N} / \mathrm{V}) \sigma_{\mathrm{s}}{ }^{3}=0.8$ which is a typical value used for several MD simulation studies for transport coefficients of the LJ model fluid. ${ }^{30}$ The temperature of the system is chosen as $40.33 \mathrm{~K}$ for comparison with the mesoscopic solvent cases ${ }^{17,27}$ and $\mathrm{T}^{*}=\mathrm{kT} / \varepsilon=1 / 3$.

After a full equilibration of the solvent-only system, two BPs with various sizes and fixed distances between two BPs are introduced such that the particle pair is oriented along the $\mathrm{Z}$ axis with $B P$ centers at $-R_{12} / 2$ and $R_{12} / 2$. The mass of a BP, $M$, is infinity and several MD simulation methods are employed for the treatment of the infinite mass limit. ${ }^{22,23}$ In the beginning of each MD simulation, the value of $\sigma_{\mathrm{B}}$ is increased gradually from zero to $\sigma_{\mathrm{B}}$. The distances between two BPs are chosen to lie (1) $\mathrm{R}_{12}=0.7,0.9,0.11,0.13,0.16,0.19,0.22$, and $0.25 \mathrm{~nm}$ for $\sigma_{B}=0.3 \mathrm{~nm},(2) \mathrm{R}_{12}=0.15,0.17,0.19,0.21,0.24$, and 0.27 $\mathrm{nm}$ for $\sigma_{\mathrm{B}}=0.7 \mathrm{~nm}$, and (3) $\mathrm{R}_{12}=0.21,0.23,0.25,0.27,0.29$, and $0.32 \mathrm{~nm}$ for $\sigma_{B}=1.0 \mathrm{~nm}$. For the systems of $\sigma_{B}=0.3 \mathrm{~nm}$ and $R_{12} \geq 1.9 \mathrm{~nm}$, those of $\sigma_{B}=0.7 \mathrm{~nm}$ and $R_{12} \geq 1.9 \mathrm{~nm}$, and those of $\sigma_{\mathrm{B}}=1.0 \mathrm{~nm}$, the simulation box was changed to a rectangular shape where the length along $\mathrm{z}, \mathrm{L}_{\mathrm{z}}$, was increased due to the periodic boundary condition and those along $\mathrm{x}$ and $\mathrm{y}, \mathrm{L}_{\mathrm{x}}$ and $\mathrm{L}_{\mathrm{x}}$, decreased as $5.0 \mathrm{~nm}$ to keep the number density constant. In order to maintain a constant value of the pressure, 
the volume of the box was slightly increased by an amount that corresponds the excess volume occupied by two BPs and so the length of the cubic and rectangular simulation box for the system is redefined by $\left(\mathrm{N} / \mathrm{L}_{\mathrm{x}} \cdot \mathrm{L}_{\mathrm{y}} \cdot \mathrm{L}_{\mathrm{z}}\right) \sigma_{\mathrm{B}}{ }^{3}+\left(2 / \mathrm{L}_{\mathrm{x}} \cdot \mathrm{L}_{\mathrm{y}} \cdot \mathrm{L}_{\mathrm{z}}\right) \sigma_{\mathrm{s}}{ }^{3}=0.8$.

Long range corrections to the energy, pressure, etc. due to the potential truncation were included in these properties by assuming that the pair distribution function was uniform beyond the cutoff distance. ${ }^{31}$ The equations of motion were solved using the velocity Verlet algorithm ${ }^{32}$ with a time step of $2.0 \times$ $10^{-15}$ second. The systems were fully equilibrated and the equilibrium properties were averaged over five blocks of 1,000,000 time steps of 10 different initial configurations. The configurations of two BP particles were stored every five time steps for further analysis.

Shear viscosity of the $\mathrm{LJ}$ solvent only is calculated by the modified Green-Kubo formula for better statistical accuracy: ${ }^{33}$

$$
\eta=\frac{\mathrm{V}}{\mathrm{kT}} \int_{0}^{\infty} \mathrm{dt} \sum_{\mathrm{i}}<\mathrm{P}_{\mathrm{i} \alpha \beta}(0) \mathrm{P}_{\mathrm{i} \alpha \beta}(\mathrm{t})>
$$

where

$$
\mathrm{P}_{\mathrm{i} \alpha \beta}(\mathrm{t})=\frac{1}{\mathrm{~V}}\left[\mathrm{~m} \mathrm{v}_{\mathrm{i} \alpha}(\mathrm{t}) \mathrm{v}_{\mathrm{i} \beta}(\mathrm{t})+\sum_{\mathrm{j} \neq \mathrm{i}} \mathrm{r}_{\mathrm{ij \alpha}}(\mathrm{t}) \mathrm{f}_{\mathrm{ij} \beta}(\mathrm{t})\right]
$$

with $\alpha \beta=x y, x z, y x, y z, z x$, and $z y$.

The fixed-particle friction tensor for two Brownian particles (BPs) can be obtained from the time integral of the force auto-correlation function as

$$
\zeta^{\alpha \beta}\left(\mathrm{R}_{12}\right)=\frac{1}{\mathrm{kT}} \int_{0}^{\infty} \mathrm{dt} \lim _{\mathrm{M} \rightarrow \infty}<\mathrm{f}_{\alpha}(0) \cdot \mathrm{f}_{\beta}(\mathrm{t})>,
$$

where $\mathrm{f}_{\alpha}(\mathrm{t})=\mathrm{F}_{\alpha}(\mathrm{t})-<\mathrm{F}_{\alpha}>$ with $\mathrm{F}_{\alpha}(\mathrm{t})$ the force on the BP $\alpha, \mathrm{k}$ is Boltzmann's constant and $\mathrm{T}$ is the absolute temperature. Isotropy yields the relations, $\zeta_{\mathrm{zz}}^{11}=\zeta_{\mathrm{zz}}^{22}$ and $\zeta_{\mathrm{xx}}^{11}=\zeta_{\mathrm{xx}}^{22}=\zeta_{\mathrm{yy}}^{11}=\zeta_{\mathrm{yy}}^{22}$ for the self-friction terms, and $\zeta_{\mathrm{zz}}^{12}=\zeta_{\mathrm{zz}}^{21}$ and $\zeta_{\mathrm{xx}}^{12}=\zeta_{\mathrm{xx}}^{21}=\zeta_{\mathrm{yy}}^{12}=$ $\zeta_{\mathrm{yy}}^{21}$ for the cross friction terms.

A number of different friction tensor can be defined for this two BPs problem since the friction tensor in Eq.(4) is labeled by particle indices and spatial components. In view of the symmetry properties noted above, we define the relative friction components $\zeta_{\mathrm{zz}}^{\alpha \beta}\left(\mathrm{R}_{12}\right)$ and $\zeta_{\mathrm{xx}}^{\alpha \beta}\left(\mathrm{R}_{12}\right)$ by

$$
\begin{aligned}
& \zeta^{(-)}\left(\mathrm{R}_{12}\right)=\frac{1}{\mathrm{kT}} \int_{0}^{\infty} \mathrm{dt} \lim _{\mathrm{M} \rightarrow \infty}<\mathrm{f}_{12}(0) \cdot \mathrm{f}_{12}(\mathrm{t})>, \\
& =2\left[\zeta^{11}\left(\boldsymbol{R}_{12}\right)-\zeta^{12}\left(\boldsymbol{R}_{12}\right)\right]
\end{aligned}
$$

where $f_{12}(t)$ is defined by

$$
f_{12}=F_{1}-F_{2}
$$

\section{Results and Discussion}

In the definition of the friction coefficient three limit pro- cedures are involved; the long time limit $(\mathrm{t} \rightarrow \infty)$; the thermodynamic limit $(\mathrm{N} \rightarrow \infty)$; and the infinite mass limit $(\mathrm{M} \rightarrow \infty){ }^{9}$ If we first consider the infinite time limit the resulting friction coefficient is zero. The only way to have a non-zero value for the friction coefficient is by first taking $\mathrm{M} \rightarrow \infty .{ }^{9}$ In the thermodynamic limit $\mathrm{N} \rightarrow \infty$, the projected and unprojected force autocorrelation functions are the same $\mathrm{e}^{34}$ and the equation (4) is possible. In order to calculate the friction coefficients between the BPs from Eqs.(4) and (5), the mass of the BP, M, becomes infinity, or the BPs are fixed in space using a holonomic constraint method. ${ }^{35}$ While the MD simulation by using an infinite mass violates the equation of motion since the BPs never move with the force on it, the constraint method MD simulation returns the BPs back to their original positions with zero velocities, and trajectories by both MD simulations are not the same. However, it is found that the momenta of the whole system carried out by both the infinite mass and the constraint method MD simulations are not conserved, because the momentum of the $\mathrm{BP}$ is not well defined with zero velocity and infinite mass. A reasonable trick to bypass this difficulty is to put the mass of the BP as $10^{90} \mathrm{~g} / \mathrm{mol}$, and in this case the momentum of the system is conserved: The magnitude of the mass of the BP is on the order of 90 and its velocity is on the order of -90 , but its momentum has a finite value and is equal to the negative of the total momentum of the solvent particles. ${ }^{22,23}$

Figure 1 shows the MD simulation results for the two-particle friction coefficients between two BPs with $\sigma_{\mathrm{B}}=0.3 \mathrm{~nm}$ as a function of the inter-particle separation, $\mathrm{R}_{12}$. We have also carried out a set of calculations for $\sigma_{\mathrm{B}}=0.7$ and $1.0 \mathrm{~nm}$ but the results show a very similar trend of the case of $\sigma_{\mathrm{B}}=0.3 \mathrm{~nm}$. The friction coefficients were obtained from the extrapolation of the time-dependent friction to $t=0$ as discussed in our earlier investigation of the friction coefficient for a single BP. ${ }^{17,22,23}$ The components of the friction normal to the intermolecular axis, $\zeta_{\mathrm{xx}}^{11}$ and $\zeta_{\mathrm{xx}}^{12}$, are seen to be almost independent of $\mathrm{R}_{12}$.

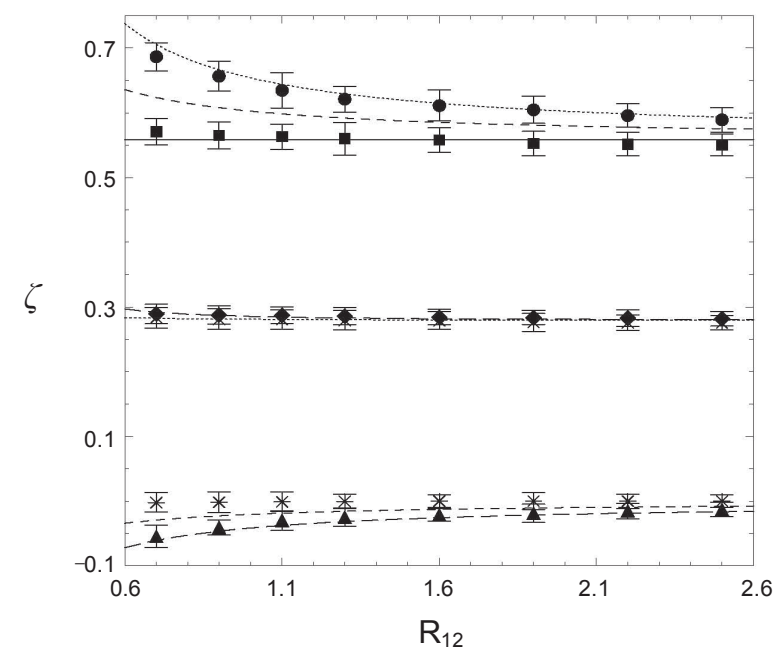

Figure 1. Friction coefficients (kg/mol.ps) for $\sigma=0.3 \mathrm{~nm}$ as a function of $\mathrm{R}_{12}(\mathrm{~nm}) \cdot \bullet: \zeta_{\mathrm{zz}}^{(-)}, \mathbf{m}: \zeta_{\mathrm{xx}}^{(-)}, \bullet: \zeta_{\mathrm{zz}}^{11}, \mathrm{x}: \zeta_{\mathrm{xx}}^{11}, *: \zeta_{\mathrm{xx}}^{12}$, and $\boldsymbol{\Delta}: \zeta_{\mathrm{zz}}^{12}$, respectively. The solid line indicates twice the single particle friction, $2 \cdot \zeta_{0}=0.558 \mathrm{~kg} / \mathrm{mol} \cdot \mathrm{ps}$, and the other lines from top are the analytic results for $\zeta_{\mathrm{zz}}^{(-)}, \zeta_{\mathrm{xx}}^{(-)}, \zeta_{\mathrm{zz}}^{11}, \zeta_{\mathrm{xx}}^{11}, \zeta_{\mathrm{xx}}^{12}$, and $\zeta_{\mathrm{zz}}^{12}$, respectively. 
According to Eq. (4),

$$
\zeta_{\mathrm{xx}}^{11}=\frac{1}{\mathrm{kT}} \int_{0}^{\infty} \mathrm{dt} \lim _{\mathrm{M} \rightarrow \infty}<\mathrm{f}_{1 \mathrm{x}}(0) \mathrm{f}_{1 \mathrm{x}}(\mathrm{t})>\approx \zeta^{0}
$$

and

$$
\zeta_{\mathrm{xx}}^{12}=\frac{1}{\mathrm{kT}} \int_{0}^{\infty} \mathrm{dt} \lim _{\mathrm{M} \rightarrow \infty}<\mathrm{f}_{1 \mathrm{x}}(0) \mathrm{f}_{2 \mathrm{x}}(\mathrm{t})>\approx 0
$$

since the BP pair is oriented along the $\mathrm{z}$ axis. On the other hand, the components parallel to this axis, $\zeta_{\mathrm{zz}}^{11}$ and $\zeta_{\mathrm{zz}}^{12}$, increase as the inter-particle separation decreases since the forces $f_{1 z}$ and $f_{2 z}$ are coupled through the solvent particles between BPs. This trend is seen even more clearly in the relative friction where $\zeta_{\mathrm{xx}}^{(-)}$is nearly independent of $\mathrm{R}_{12}$ and equal to its asymptotic value of twice the single particle friction coefficient according to Eq.(5) since $\zeta_{\mathrm{xx}}^{11} \approx \zeta^{0}$ and $\zeta_{\mathrm{xx}}^{12} \approx 0$. The parallel component, $\zeta_{\mathrm{zz}}^{(-)}$, increases as $\mathrm{R}_{12}$ decreases.

The overall behavior of the two-particle friction coefficients between two BPs in a LJ solvent as a function of the inter-particle separation, $\mathrm{R}_{12}$, obtained from the MD simulations is almost the same with that in a mesoscopic solvent ${ }^{27}$ even though the absolute values of friction coefficients are very different. For example, the single particle friction coefficients, $\zeta^{-0}$, in a LJ solvent are $0.279,0.545$, and $0.791 \mathrm{~kg} / \mathrm{mol} \cdot \mathrm{ps}$ for $\sigma_{\mathrm{B}}=0.3,0.7$, and $1.0 \mathrm{~nm}$, respectively, while the corresponding $\zeta^{-0}$ in a mesoscopic solvent are $0.604,1.62$, and 2.65, with different values of viscosity $\eta$ for the solvent-only system, which is discussed below.

Next we have chosen to contrast our simulation results with the predictions of a very simple hydrodynamic model simply to gauge the rough magnitudes of hydrodynamic effects. If we assume that hydrodynamic interactions between the particles are given by Oseen interactions, the two-particle friction tensor takes the form, ${ }^{40}$

$$
\zeta\left(\mathrm{R}_{12}\right)=\zeta_{0}\left[\mathrm{I}+\zeta_{0} \mathrm{~T}\left(\mathrm{R}_{12}\right)\right]^{-1},
$$

where $\zeta_{0}$ is the one-particle friction coefficient and $\mathrm{T}\left(\mathrm{R}_{12}\right)$ is the Oseen tensor,

$$
\mathrm{T}_{\alpha \beta}\left(\mathrm{R}_{12}\right)=\frac{\left(1-\delta_{\alpha \beta}\right)}{8 \pi \eta \mathrm{R}_{12}}\left(1+\hat{\mathrm{R}}_{12} \hat{\mathrm{R}}_{12}\right) .
$$

Here $\hat{\mathrm{R}}_{12}$ is a unit vector along the inter-particle (z) axis. Taking the inverse of the matrix in Eq. (9) we find, ${ }^{24}$

$$
\begin{aligned}
& \zeta_{\mathrm{zz}}^{11}\left(\mathrm{R}_{12}\right)=\frac{\zeta_{0}}{1-\left[2 h\left(\mathrm{R}_{12}\right)\right]^{2}}, \quad \zeta_{\mathrm{xx}}^{11}\left(\mathrm{R}_{12}\right)=\frac{\zeta_{0}}{1-\left[h\left(\mathrm{R}_{12}\right)\right]^{2}}, \\
& \zeta_{\mathrm{zz}}^{12}\left(\mathrm{R}_{12}\right)=\frac{-2 \zeta_{0} h\left(\mathrm{R}_{12}\right)}{1-\left[2 h\left(\mathrm{R}_{12}\right)\right]^{2}}, \quad \zeta_{\mathrm{xx}}^{12}\left(\mathrm{R}_{12}\right)=\frac{-\zeta_{0} h\left(\mathrm{R}_{12}\right)}{1-\left[h\left(\mathrm{R}_{12}\right)\right]^{2}} .
\end{aligned}
$$

Furthermore, according to Eq. (5),
$\zeta_{\mathrm{zz}}^{(-)}\left(\mathrm{R}_{12}\right)=\frac{2 \zeta_{0}}{1-2 h\left(\mathrm{R}_{12}\right)}, \quad \zeta_{\mathrm{xx}}^{(-)}\left(\mathrm{R}_{12}\right)=\frac{2 \zeta_{0}}{1-h\left(\mathrm{R}_{12}\right)}$

where $h\left(\mathrm{R}_{12}\right)=\zeta_{0} / 8 \pi \eta \mathrm{R}_{12}$ with $\eta$ the viscosity for the solvent-only system.

The viscosity for the solvent-only system of $\mathrm{N}=32,000$ Lennard-Jones (LJ) particles of mass $\mathrm{m}=3.995 \mathrm{~g} / \mathrm{mol}$ in the cubic simulation box of length $L=6.84 \mathrm{~nm}\left(\rho=100 \mathrm{~nm}^{-3}\right)$ interacting through the WCA potential with $\mathrm{LJ}$ parameters $\sigma_{\mathrm{s}}=$ $0.2 \mathrm{~nm}$ and $\varepsilon=1.00604 \mathrm{~kJ} / \mathrm{mol}$ at $\mathrm{T}=40.33 \mathrm{~K}$ obtained from Eq.(2) is $\eta=2.53 \pm 0.02 \mathrm{mP}\left(10^{-4} \mathrm{~kg} / \mathrm{m} \cdot \mathrm{s}\right)$ and the reduced viscosity $\eta^{*}=\eta \sigma_{\mathrm{s}}^{2}(\mathrm{~m} \varepsilon)^{1 / 2}=12.2$. Comparing with the previous mesoscopic MD simulation study ${ }^{17}$ where $\rho=2035.42 \mathrm{~nm}^{-3}$ with same $\mathrm{m}$ and $\mathrm{T}$ but $\sigma_{\mathrm{s}}=0$, the corresponding viscosity was $\eta=4.70 \mathrm{mP}$.

For comparison, in Figure 1 we also plot these expressions for the friction tensor components, Eqs.(11)-(13). The simple hydrodynamic model estimates a very small difference from the single particle friction coefficients, $\zeta^{-}$, for the self-friction terms at all inter-particle separations while the cross friction terms vary dramatically at small inter-particle separations. The agreement of the friction results for $\zeta_{\mathrm{zz}}^{11}, \zeta_{\mathrm{xx}}^{11}$, and $\zeta_{\mathrm{zz}}^{12}$ between the MD simulation and the simple hydrodynamic model is generally good but that for $\zeta_{\mathrm{xx}}^{12}$ is very poor. Our MD simulations show that $\zeta_{\mathrm{xx}}^{12}$ varies much more weakly with inter-particle separation than the predictions of the simple hydrodynamic model. While the disagreement of $\zeta_{\mathrm{xx}}^{12}$ results in the disagreement of the relative friction, $\zeta_{\mathrm{xx}}^{(-)}$, which is equal to its asymptotic value of twice the single particle friction coefficient, the agreement of the other relative friction, $\zeta_{\mathrm{zz}}^{(-)}$, is quite good. There are deviations for the relative frictions at small inter-particle separations as might be expected since the simple hydrodynamic approximations will be poorest at these distances.

In summary, we presented a molecular dynamics (MD) simulation study of friction behavior between two very massive Brownian particles (BPs) oriented along the $\mathrm{z}$ axis with BP centers at $-\mathrm{R}_{12} / 2$ and $\mathrm{R}_{12} / 2$ in a Lennard-Jones solvent as a function of the inter-particle separation, $\mathrm{R}_{12}$. In order to fix the BPs in space an MD simulation method with the mass of the $\mathrm{BP}$ as $10^{90} \mathrm{~g} / \mathrm{mol}^{22,23}$ was employed in which the total momentum of the system was conserved. The cross friction coefficients of $\mathrm{x}$ - and $\mathrm{y}$-components are nearly insensitive to $\mathrm{R}_{12}$ but that of $\mathrm{z}$-component varies with $\mathrm{R}_{12}$ in good accord with the simple hydrodynamic approximation. On the other hand, the self-friction coefficients are estimated as a very small difference from the single particle friction coefficients, $\zeta^{0}$, at all inter-particle separations which agrees with the simple hydrodynamic approximation. Consequently $\zeta_{\mathrm{xx}}^{(-)}$is nearly independent of $\mathrm{R}_{12}$ and equal to its asymptotic value of twice the single particle friction coefficient, and the other relative friction, $\zeta_{\mathrm{zz}}^{-()}$, is in good agreement with the simple hydrodynamic approximation.

Acknowledgments. This research was supported by Kyungsung University Research Grants in 2010.

\section{References}

1. Lebowitz, J. L.; Rubin, E. Phys. Rev. 1963, 131, 2381. 
2. Résibois, P.; Davis, H. T. Physica 1964, 30, 1077.

3. Lebowitz, J. L.; Résibois, P. Phys. Rev. 1965, 139, 1101.

4. Cukier, R. I.; Deutch, J. M. Phys. Rev. 1969, 177, 240.

5. Mazur P.; Oppenheim, I. J. Phys. Soc. Japan Suppl. 1969, 26, 35.

6. Mazur, P.; Oppenheim, I. Physica (Amsterdam) 1970, 50, 241.

7. Albers, J.; Deutch, J. M.; Oppenheim, I. J. Chem. Phys. 1971, 54, 3541.

8. Brey, J. J.; Gómez Ordóñez, J. J. Chem. Phys. 1982, 76, 3260.

9. Español, P.; Zúñiga, I. J. Chem. Phys. 1993, 98, 574.

10. Bocquet, L.; Hansen, J.-P.; Piasecki, J. J. Stat. Phys. 1994, 76, 527.

11. Heyes, D. M.; Nuevo, M. J.; Morales, J. J.; Branka, A. C. J. Phys.; Condens. Matter 1998, 10, 10159.

12. McDonough, A.; Russo, S. P.; Snook, I. K. Phys. Rev. E 2001, 63, 026109.

13. Schmidt J. R.; Skinner, J. L. J. Chem. Phys. 2003, 119, 8062.

14. Ould-Kaddour, F.; Levesque, D. Phys. Rev. E 2001, 63, 011205.

15. Willeke, M. Mol. Phys. 2003, 101, 1123.

16. Ould-Kaddour, F.; Levesque, D. J. Chem. Phys. 2003, 118, 7888

17. Lee, S. H.; Kapral, R. J. Chem. Phys. 2004, 121, 11163.

18. Sokolovskii, R. O.; Thachuk, M.; Patey, G. N. J. Chem. Phys. 2006, 125,204502

19. Cappelezzo, M.; Capellari, C. A.; Pezzin, S. H.; Coelho, L. A. F. J. Chem. Phys. 2007, 126, 224516

20. Funazukuri, T.; Kong, C. Y.; Kagei, S. J. Supercritical Fluids 2008 , $46,280$.

21. Harris, K. R. J. Chem. Phys. 2009, 131, 054503.

22. Lee, S. H. Bull. Korean Chem. Soc. 2010, 31, 959.

23. Lee, S. H. Theor. Chem. Acc., published online: 04 May 2010,
DOI:10.1007/s00214-010-0757-z.

24. Deutch, J. M.; Oppenheimer, I. J. Chem. Phys. 1971, 54, 3547.

25. Mazo, R. M. J. Stat. Phys. 1969, 1, 559.

26. Zwanzig, R. W. J. Res. Natl. Bur. Std. 1964, 68, 143.

27. Lee, S. H.; Kapral, R. J. Chem. Phys. 2005, 122, 214916.

28. Malevanets, A.; Kapral, R. J. Chem. Phys. 1999, 110, 8605; ibid 2000, 112, 7260; ibid, Lect. Notes Phys. 2004, 640, 116.

29. Weeks, J. D.; Chandler, D.; Andersen, H. C. J. Chem. Phys. 1971, $54,5237$.

30. Meier, M.; Laesecke, A.; Kabelac, S. J. Chem. Phys. 2004, 121, 3671; ibid 2004, 121, 9526; ibid 2005, 122, 014513.

31. Allen, M. P.; Tildesley, D. J. Computer Simulation of Liquids; Oxford Univ Press: Oxford, 1987

32. Swope, W. C.; Andersen, H. C.; Berens, P. H.; Wilson, K. R. J. Chem. Phys. 1982, 76, 637.

33. Lee, S. H. Bull. Korean Chem. Soc. 2007, 28, 1371

34. Kubo, R. J. Phys. Soc. Jpn. 1957, 12, 570; Kubo, R. Rep. Prog. Phys. 1966, 29, 255; Kubo, R. In Many-Body Problems, The Fluctuation Dissipation Theorem, Parry, W. E. et al., Eds.; Benjamin: New York, 1969; p 235.

35. Ryckaert, J. P.; Ciccotti, G.; Berendsen, H. J. J. Comp. Phys. 1977, $23,327$.

36. Stimson, M.; Jeffery, G. B. Proc. Roy. Soc. (London) 1926, 111, 110 .

37. Wacholder, E.; Weihs, D. Chem. Eng. Sci. 1972, 27, 1817.

38. Felderhof, B. U. Physica A 1977, 89, 373.

39. Schmitz, R.; Felderhof, B. U. Physica A 1982, 113, 103.

40. Kirkwood, J. G.; Riseman, J. J. Chem. Phys. 1948, 16, 565. 